\title{
Binary Bell Polynomials, Hirota Bilinear Approach to Levi Equation
}

\author{
Yaning Tang*, Weijian Zai, Siqiao Tao, Qing Guan \\ Department of Applied Mathematics, Northwestern Polytechnical University, \\ Xi'an 710129, P.R. China
}

\begin{abstract}
Combining the binary Bell polynomials and Hirota method, we obtained two kinds of equivalent bilinear equations for the Levi equation. Then, we got the double Wronskian solutions of the Levi equation by virtue of one of the bilinear equations. Furthermore, we constructed the bilinear Bäcklund transformation and the Lax pair. Finally, we also derived the Darboux transformation and the infinite conservation laws of the Levi equation.
\end{abstract}

Key words: Binary Bell polynomials, Double Wronskian solutions, Bäcklund transformation and Lax pair, Darboux transformation, Infinite conservation laws

PACS: 05.45.Yv, 02.30.Jr, 03.65.Ge

\section{Introduction}

Nonlinear equations are widely used in the fields of physics, chemistry, atmospheric dynamics, life sciences and engineering sciences etc. There have been many great researches on the nonlinear equations in recent years. Particularly in soliton theory, many different approaches can be used to deal with the nonlinear equations. Inverse scattering transformation [1], Bäcklund transformation [2], Darboux transformation [3], Hirota bilinear method [4, 5] and Wronskian technique [6] are all very cogent methods. Among those methods, the bilinear method developed by Hirota is powerful for analysing soliton equations. The first step of this method is to transform the given soliton equations into bilinear forms by proper dependent variables transformation. By means of the bilinear representations for soliton equations, not only the multi-soliton solutions but also the main integrability features of these equations such as bilinear Bäcklund transformations,

*E-mail address: tyaning@nwpu.edu.cn 
Lax pairs and Darboux transformation can be derived. However, the well chosen dependent variables transformation for deriving the bilinear forms can not be obtained easily, because it relies on clever guesswork and tedious calculation. Recently, Lembert, Gilson et al. [7-10] found there exists a deep connection between the Bell polynomials and Hirota's bilinear expressions and then proposed a lucid and systematic method to overcome these difficulties. So both the bilinear forms and bilinear Bäcklund transformations can be obtained directly. Lots of studies have devoted to the application and extension of this method [11-21].

In this paper, we will study the Levi equation

$$
\begin{aligned}
& u_{t}-2 u u_{x}-2 v_{x}+u_{x x}=0 \\
& v_{t}-2(u v)_{x}-v_{x x}=0
\end{aligned}
$$

Eqs.(1.1) has been investigated in [22] for solving the the double Wronskian solutions. However, the bilinear Bäcklund transformations, Lax pair, Darboux transformation as well as the infinite conservation Laws for Eqs.(1.1) have not been studied based on the binary Bell polynomials and Hirota method.

On account of the above analysis, this paper is organized as follows. In Section 2, we will briefly introduce concepts and formulae about the binary Bell polynomials. In Section 3, we will derive two kinds of equivalent bilinear equations for Eqs.(1.1), then we will get the double Wronskian solutions of Eqs.(1.1). In Section 4, we will give the bilinear Bäcklund transformation and the corresponding Lax pair. In Section 5, we will obtain the Darboux transformation by introducing a gauge transformation. In Section 6, we also will get the infinite conservation laws of Eqs.(1.1). Finally, we will draw some conclusions.

\section{The binary Bell polynomials}

Let $f=f\left(x_{1}, \ldots, x_{l}\right)$ is a $C^{\infty}$ function of multi-variables, the definition of multi-dimensional Bell polynomials ( see $[7-10]$ for details ) reads as

$$
Y_{n_{1} x_{1}, \ldots, n_{l} x_{l}}(f) \equiv Y_{n_{1}, \ldots, n_{l}}\left(f_{r_{1} x_{1}, \ldots, r_{l} x_{l}}\right)=e^{-f} \partial_{x_{1}}^{n_{1}} \ldots \partial_{x_{l}}^{n_{l}} e^{f}
$$

where $f_{r_{1} x_{1}, \ldots, r_{l} x_{l}}=\partial_{x_{1}}^{r_{1}} \ldots \partial_{x_{l}}^{r_{l}} f, r_{1}=0, \ldots, n_{1} ; \cdots ; r_{l}=0, \ldots, n_{l}$.

Based on the above Bell polynomials, when $l=2$ in (2.1), the binary Bell polynomials $(\mathcal{Y}$-polynomials) can be defined by

$$
\mathcal{Y}_{n_{1} x, n_{2} t}(v, w)=\left.Y_{n_{1} x, n_{2} t}(f)\right|_{f_{r_{1} x, r_{2} t}=} \begin{cases}v_{r_{1} x, r_{2}}, & r_{1}+r_{2}=\text { odd } \\ w_{r_{1} x, r_{2} t}, & r_{1}+r_{2}=\text { even }\end{cases}
$$


For example, the first few lowest order $\mathcal{Y}$-polynomials are

$$
\begin{aligned}
& \mathcal{Y}_{x, t}(v, w)=w_{x t}+v_{x} v_{t}, \\
& \mathcal{Y}_{2 x, t}(v, w)=v_{2 x, t}+w_{2 x} v_{t}+2 w_{x t} v_{x}+v_{x}^{2} v_{t}, \\
& \mathcal{Y}_{3 x}(v, w)=v_{3 x}+3 w_{2 x} v_{x}+v_{x}^{3}, \ldots
\end{aligned}
$$

The link between $\mathcal{Y}$-polynomials and the Hirota's standard bilinear expressions

$$
D_{x}^{n_{1}} D_{t}^{n_{2}} F \cdot G=\left(\partial_{x}-\partial_{x^{\prime}}\right)^{n_{1}}\left(\partial_{t}-\partial_{t^{\prime}}\right)^{n_{2}} \times\left. F(x, t) G\left(x^{\prime}, t^{\prime}\right)\right|_{x^{\prime}=x, t^{\prime}=t}
$$

is given by the identity

$$
\mathcal{Y}_{n_{1} x, n_{2} t}(v=\ln F / G, w=\ln F G)=(F G)^{-1} D_{x}^{n_{1}} D_{t}^{n_{2}} F \cdot G .
$$

Particularly, in the case of $F=G$, we have

$$
\begin{aligned}
& F^{-2} D_{x}^{n_{1}} D_{t}^{n_{2}} F \cdot F=\mathcal{Y}_{n_{1} x, n_{2} t}(0, w=2 \ln F) \\
= & \begin{cases}0, & n_{1}+n_{2}=\text { odd }, \\
P_{n_{1} x, n_{2} t}(w), & n_{1}+n_{2}=\text { even, }\end{cases}
\end{aligned}
$$

which discloses that $P$-polynomials can be obtained by restricting the Bell Polynomials to even part partitions.

Moreover, there has a relationship between the binary Bell polynomials and Lax pair through the following expression

$$
\begin{aligned}
& \mathcal{Y}_{n_{1} x, n_{2} t}(v=\ln \Psi, w=v+Q) \\
= & \Psi^{-1} \sum_{a_{1}=0}^{n_{1}} \sum_{a_{2}=0}^{n_{2}}\left(\begin{array}{c}
n_{1} \\
a_{1}
\end{array}\right)\left(\begin{array}{c}
n_{2} \\
a_{2}
\end{array}\right) P_{a_{1} x, a_{2} t}(Q) Y_{\left(n_{1}-a_{1}\right) x,\left(n_{2}-a_{2}\right) t}(v=\ln \Psi),
\end{aligned}
$$

where $\Psi$ and $Q$ are functions of $x$ and $t$.

\section{Bilinear equations and double Wronskian solutions}

In this section, with the help of the binary Bell polynomials, we will give two kinds of equivalent bilinear forms of Eqs.(1.1).

Let

$$
u=p_{x}, \quad v=\frac{1}{2}\left(q_{x x}+p_{x x}\right),
$$

Eqs.(1.1) are transformed to

$$
\begin{aligned}
& p_{t}-\left(p_{x}^{2}+q_{x x}\right)=0, \\
& q_{x t}-p_{x x x}-2 p_{x} q_{x x}=0,
\end{aligned}
$$


using Eq.(3.2a), Eqs.(3.2) are further transformed to

$$
\begin{aligned}
& p_{t}-\left(p_{x}^{2}+q_{x x}\right)=0, \\
& \left(q_{x t}+p_{x} p_{t}\right)-\left(p_{x x x}+3 p_{x} q_{x x}+p_{x}^{3}\right)=0 .
\end{aligned}
$$

From the expressions (2.2), Eqs.(3.3) are equivalent to

$$
\begin{aligned}
& \mathcal{Y}_{t}(p)-\mathcal{Y}_{2 x}(p, q)=0, \\
& \mathcal{Y}_{x t}(p, q)-\mathcal{Y}_{3 x}(p, q)=0
\end{aligned}
$$

By setting

$$
p=\ln (h / f), \quad q=\ln (h f),
$$

then by using Eq.(2.3) and Eq.(2.4), the bilinear equations for Eqs.(1.1) are obtained as

$$
\begin{aligned}
& \left(D_{t}-D_{x}^{2}\right) h \cdot f=0, \\
& \left(D_{x} D_{t}-D_{x}^{3}\right) h \cdot f=0,
\end{aligned}
$$

where $h$ and $f$ are the functions of $x$ and $t$.

Based on the above bilinear equations, we can get their double Wronskian determinant solutions, but the process for proving the existing of Wronskian solutions will be very difficult because Eq.(3.6b) is third-order on $h$ and $f$. In order to make the problem not too difficult, we may first reduce Eq.(3.6b) to two second-order bilinear equations on $h$ and $f$ by adding an auxiliary variable.

Theorem 3.1 Let

$$
f^{2} \ln (f)_{x x}=-g h
$$

where $g$ is an auxiliary variable ( which is the function of $x$ and $t$ ), then Eqs.(3.6) are equivalent to the following three second-order bilinear equations

$$
\begin{aligned}
& \left(D_{t}-D_{x}^{2}\right) h \cdot f=0, \\
& D_{x}^{2} f \cdot f=-2 g h, \\
& \left(D_{t}+D_{x}^{2}\right) g \cdot f=0,
\end{aligned}
$$

Proof: From Eq.(3.7) and Eq.(2.3), we can get Eq.(3.8b) directly, hence we only need to prove Eq.(3.8c).

Taking the derivative with respect to $x$ on both sides of Eq.(3.2b), we have

$$
q_{x x t}-p_{x x x x}-2\left(p_{x} q_{x x}\right)_{x}=0 .
$$


Based on the transformation (3.5) and Eq.(3.7), we can get $q_{x x}=p_{x x}-\frac{2 g}{f} \cdot \frac{h}{f}$, substituting it into Eq.(3.9), we have

$$
p_{x x t}-\left(\frac{2 g}{f} \cdot \frac{h}{f}\right)_{t}-p_{x x x x}-2\left(p_{x} p_{x x}\right)_{x}+2\left(p_{x} \frac{2 g}{f} \cdot \frac{h}{f}\right)_{x}=0,
$$

using $p_{x x t}-2\left(p_{x} p_{x x}\right)_{x}-p_{x x x x}=-\left(\frac{2 g}{f} \cdot \frac{h}{f}\right)_{x x}$ and $p_{x}=\left(\frac{h}{f}\right)_{x} \cdot \frac{f}{h}$, Eq.(3.10) is transformed to

$$
-\left(\frac{g}{f}\right)_{x x} \cdot \frac{h}{f}+\frac{g}{f} \cdot\left(\frac{h}{f}\right)_{x x}-\left(\frac{h}{f}\right)_{t} \cdot \frac{g}{f}-\frac{h}{f} \cdot\left(\frac{g}{f}\right)_{t}=0 .
$$

From Eq.(3.3a) and the transformation (3.5), we can get

$$
\left(\frac{h}{f}\right)_{t}=\frac{h}{f}\left(\ln \left(\frac{h}{f}\right)\right)_{x}^{2}+\frac{h}{f}\left(\ln \left(\frac{h}{f}\right)\right)_{x x}-\frac{h}{f} \cdot \frac{2 g h}{f^{2}},
$$

substituting it into Eq.(3.11), we obtain

$$
\left(\frac{g}{f}\right)_{x x}+\frac{g}{f} \frac{2 g h}{f^{2}}+\left(\frac{g}{f}\right)_{t}=0
$$

That is just Eq.(3.8c) by using Eq.(2.3) and Eq.(3.8b).

Let we give the double Wronskian solutions based on Eqs.(3.8).

Theorem 3.2 Eqs.(3.8) have the double Wronskian solutions

$$
h=-2 W^{N, M+2}(\Phi ; \Psi), \quad f=W^{N+1, M+1}(\Phi ; \Psi), \quad g=2 W^{N+2, M}(\Phi ; \Psi),
$$

where the entries $\Phi, \Psi(j=1, \ldots, N)$ satisfy the following linear differential conditions :

$$
\Phi_{i, x}=-k_{i} \Phi_{i}, \quad \Phi_{i, t}=-2 \Phi_{i, x x}, \quad \Psi_{i, x}=k_{i} \Psi_{i}, \quad \Psi_{i, t}=2 \Psi_{i, x x}, \quad 1 \leq i \leq N,
$$

and the double Wronskian is defined as $[5,6]$

$$
W^{N, M}(\Phi ; \Psi)=\operatorname{det}\left(\Phi, \partial_{x} \Phi, \ldots, \partial_{x}^{N-1} \Phi ; \Psi, \partial_{x} \Psi, \ldots, \partial_{x}^{N-1} \Psi\right)
$$

with $\Phi=\left(\Phi_{1}, \Phi_{2}, \ldots, \Phi_{N+M+2}\right)^{T}, \Psi=\left(\Psi_{1}, \Psi_{2}, \ldots, \Psi_{N+M+2}\right)^{T}$.

Proof: In the following, we use the abbreviated notation of Freeman and Nimmo for the Wronskian and its derivatives [23-26], then Eqs. (3.13) become

$$
h=-2|\widehat{N-1}, \widehat{M+1}|, \quad f=|\widehat{N}, \widehat{M}|, \quad g=2|\widehat{N+1}, \widehat{M-1}|,
$$


we have

$$
\begin{aligned}
f_{x}= & |\widehat{N-1}, N+1, \widehat{M}|+|\widehat{N}, \widehat{M-1}, M+1|, \\
f_{x x}= & |\widehat{N-2}, N, N+1, \widehat{M}|+|\widehat{N-1}, N+2, \widehat{M}|+2|\widehat{N-1}, N+1, \widehat{M-1}, M+1| \\
& +|\widehat{N}, \widehat{M-2}, M, M+1|+|\widehat{N}, \widehat{M-1}, M+2|, \\
f_{t}= & 2\{|\widehat{N-2}, N, N+1, \widehat{M}|-|\widehat{N-1}, N+2, \widehat{M}|-|\widehat{N}, \widehat{M-2}, M, M+1| \\
& +|\widehat{N}, \widehat{M-1}, M+2|\}, \\
h_{x}= & -2\{|\widehat{N-2}, N, \widehat{M+1}|+|\widehat{N-1}, \widehat{M}, M+2|\}, \\
h_{x x}= & -2\{|\widehat{N-3}, N-1, N, \widehat{M+1}|+|\widehat{N-2}, N+1, \widehat{M+1}|+2|\widehat{N-2}, N, \widehat{M}, M+2| \\
& +|\widehat{N-1}, \widehat{M-1}, M+1, M+2|+|\widehat{N-1}, \widehat{M}, M+3|\}, \\
h_{t}= & -2\{2|\widehat{N-3}, N-1, N, \widehat{M+1}|-2|\widehat{N-2}, N+1, \widehat{M+1}| \\
& -2|\widehat{N-1}, \widehat{M-1}, M+1, M+2|+2|\widehat{N-1}, \widehat{M}, M+3|\},
\end{aligned}
$$

so,

$$
\begin{aligned}
f & \left.h_{t}-h_{x x}\right) \\
= & -2|\widehat{N}, \widehat{M}|\{|\widehat{N-3}, N-1, N, \widehat{M+1}|-3|\widehat{N-2}, N+1, \widehat{M+1}| \\
& -2|\widehat{N-2}, N, \widehat{M}, M+2|-3|\widehat{N-1}, \widehat{M-1}, M+1, M+2|+|\widehat{N-1}, \widehat{M}, M+3|\} \\
= & -2|\widehat{N}, \widehat{M}|\{|\widehat{N-3}, N-1, N, \widehat{M+1}|+|\widehat{N-2}, N+1, \widehat{M+1}| \\
& -2|\widehat{N-2}, N, \widehat{M}, M+2|+|\widehat{N-1}, \widehat{M-1}, M+1, M+2|+|\widehat{N-1}, \widehat{M}, M+3| \\
& -4|\widehat{N-2}, N+1, \widehat{M+1}|-4|\widehat{N-1}, \widehat{M-1}, M+1, M+2|\}, \\
- & h\left(f_{t}+f_{x x}\right) \\
= & -2|\widehat{N-1}, \widehat{M+1}|\{-3|\widehat{N-2}, N, N+1, \widehat{M}|+|\widehat{N-1}, N+2, \widehat{M}| \\
& -2|\widehat{N-1}, N+1, \widehat{M-1}, M+1|+|\widehat{N}, \widehat{M-2}, M, M+1|-3|\widehat{N}, \widehat{M-1}, M+2|\} \\
= & -2|\widehat{N-1}, \widehat{M+1}|\{|\widehat{N-2}, N, N+1, \widehat{M}|+|\widehat{N-1}, N+2, \widehat{M}| \\
& -2|\widehat{N-1}, N+1, \widehat{M-1}, M+1|+|\widehat{N}, \widehat{M-2}, M, M+1|+|\widehat{N}, \widehat{M-1}, M+2| \\
& -4|\widehat{N-2}, N, N+1, \widehat{M}|-4|\widehat{N}, \widehat{M-1}, M+2|\}, \\
2 & h_{x} f_{x}=-4\{|\widehat{N-1}, N+1, \widehat{M}|+|\widehat{N}, \widehat{M-1}, M+1|\} . \\
& \quad\{\widehat{N-2}, N, \widehat{M+1}|+| \widehat{N-1}, \widehat{M}, M+2 \mid\} .
\end{aligned}
$$

Noting

$$
|\widehat{N}, \widehat{M}|\left[\sum_{j=1}^{N+M+2} k_{j}\right]^{2}|\widehat{N}, \widehat{M}|=\left\{\left[\sum_{j=1}^{N+M+2} k_{j}\right]|\widehat{N}, \widehat{M}|\right\}^{2}
$$

and

$$
|\widehat{N}, \widehat{M}|\left[\sum_{j=1}^{N+M+2} k_{j}\right]^{2}|\widehat{N-1}, \widehat{M+1}|=\left\{\left[\sum_{j=1}^{N+M+2} k_{j}\right]|\widehat{N}, \widehat{M}|\right\}\left\{\left[\sum_{j=1}^{N+M+2} k_{j}\right]|\widehat{N-1}, \widehat{M+1}|\right\}
$$




$$
=|\widehat{N-1}, \widehat{M+1}|\left[\left[\sum_{j=1}^{N+M+2} k_{j}\right]^{2}|\widehat{N}, \widehat{M}|,\right.
$$

we get

$$
\begin{aligned}
& |\widehat{N}, \widehat{M}|(|\widehat{N-2}, N, N+1, \widehat{M}|+|\widehat{N-1}, N+2, \widehat{M}|-2|\widehat{N-1}, N+1, \widehat{M-1}, M+1| \\
& +|\widehat{N}, \widehat{M-2}, M, M+1|+|\widehat{N}, \widehat{M-1}, M+2|) \\
& =(|\widehat{N-1}, N+1, \widehat{M}|-|\widehat{N}, \widehat{M-1}, M+1|)^{2},
\end{aligned}
$$

and

$$
\begin{aligned}
\mid & \widehat{N-1}, \widehat{M+1} \mid\{|\widehat{N-2}, N, N+1, \widehat{M}|+|\widehat{N-1}, N+2, \widehat{M}| \\
- & 2|\widehat{N-1}, N+1, \widehat{M-1}, M+1|+|\widehat{N}, \widehat{M-2}, M, M+1|+|\widehat{N}, \widehat{M-1}, M+2|\} \\
= & |\widehat{N}, \widehat{M}|\{|\widehat{N-3}, N-1, N, \widehat{M+1}|+|\widehat{N-2}, N+1, \widehat{M+1}|-2|\widehat{N-2}, N, \widehat{M}, M+2| \\
& +|\widehat{N-1}, \widehat{M-1}, M+1, M+2|+|\widehat{N-1}, \widehat{M}, M+3|\} \\
= & \{|\widehat{N-1}, N+1, \widehat{M}|-|\widehat{N}, \widehat{M-1}, M+1|\}\{|\widehat{N-2}, N, \widehat{M+1}|-|\widehat{N-1}, \widehat{M}, M+2|\},
\end{aligned}
$$

then, utilizing the identities (3.16), by tedious calculus,

$$
\begin{aligned}
\left(D_{t}-D_{x}^{2}\right) h \cdot f \\
=f\left(h_{t}-h_{x x}\right)-h\left(f_{t}+f_{x x}\right)+2 h_{x} f_{x} \\
=8|\widehat{N}, \widehat{M}|\{|\widehat{N-2}, N+1, \widehat{M+1}|+|\widehat{N-1}, \widehat{M-1}, M+1, M+2|\} \\
\quad+8|\widehat{N-1}, \widehat{M+1}|\{|\widehat{N-2}, N, N+1, \widehat{M}|+|\widehat{N}, \widehat{M-1}, M+2|\} \\
\quad-8\{|\widehat{N-1}, N+1, \widehat{M}||\widehat{N-2}, N, \widehat{M+1}|+|\widehat{N}, \widehat{M-1}, M+1||\widehat{N-1}, \widehat{M}, M+2|\} .
\end{aligned}
$$

According to Lemma 1 in [25], it is readily checked the above expression is equal to zero, which shows that Eq.(3.8a) is satisfied. Similarly, we can verify that Eq.(3.8c) is also satisfied.

For Eq.(3.8b), using the identity (3.15) and Lemma 1 in [25], we can easily get

$$
\begin{aligned}
D_{x}^{2} f \cdot f+2 g h \\
=2\left(f f_{x x}-f_{x}^{2}+g h\right) \\
=8|\widehat{N}, \widehat{M}||\widehat{N-1}, N+1, \widehat{M-1}, M+1|-8|\widehat{N-1}, N+1, \widehat{M}||\widehat{N}, \widehat{M-1}, M+1| \\
\quad-8|\widehat{N+1}, \widehat{M-1}||\widehat{N-1}, \widehat{M+1}|
\end{aligned}
$$

is also equal to zero, so the proof of Eq.(3.8b) is finished. 


\section{Bilinear Bäcklund transformation and Lax pair}

\subsection{Bilinear Bäcklund transformation}

The Bäcklund transformation (BT) [27] provides a way of constructing new solutions from known ones for the soliton equations. In this section, based on the transformation (3.5), we will obtain the bilinear BT for Eqs. (1.1).

Theorem 4.1 The bilinear BT for Eqs. (1.1) is as follows:

$$
\begin{aligned}
& \left(D_{t}-D_{x}^{2}\right) g^{\prime} \cdot g=0 \\
& \left(D_{t}-D_{x}^{2}\right) f^{\prime} \cdot f=0 \\
& D_{x} f^{\prime} \cdot g=\mu f g^{\prime} \\
& \left(D_{x}^{2}-\gamma D_{x}\right) f^{\prime} \cdot g+\mu D_{x} g^{\prime} \cdot f=0
\end{aligned}
$$

where $\gamma$ and $\mu$ are two arbitrary real constants.

Proof: Let $p^{\prime}=\ln \left(h^{\prime} / f^{\prime}\right), q^{\prime}=\ln \left(h^{\prime} f^{\prime}\right)$, and introduce the following relations:

$$
\begin{array}{ll}
v_{1}=\ln \frac{f^{\prime}}{f}, & v_{2}=\ln \frac{h^{\prime}}{h},
\end{array}
$$

combining the transformation (3.5), we have

$$
\begin{array}{ll}
p^{\prime}-p=v_{2}-v_{1}=w_{3}-w_{4}, & p^{\prime}+p=v_{3}-v_{4}=w_{2}-w_{1} \\
q^{\prime}-q=v_{1}+v_{2}=v_{3}+v_{4}, & q^{\prime}+q=w_{1}+w_{2}=w_{3}+w_{4} .
\end{array}
$$

Considering

$$
\begin{aligned}
& E_{1}=\left(p_{t}^{\prime}-p_{t}\right)-\left(p_{x}^{\prime 2}-p_{x}^{2}\right)-\left(q_{x x}^{\prime}-q_{x x}\right)=0, \\
& E_{2}=\left(q_{x t}^{\prime}-q_{x t}\right)-\left(p_{x x x}^{\prime}-p_{x x x}\right)-2\left(p_{x}^{\prime} q_{x x}^{\prime}-p_{x} q_{x x}\right)=0,
\end{aligned}
$$

we have

$$
E_{1}=\left(v_{2}-v_{1}\right)_{t}-2 v_{3, x}\left(v_{2}-v_{1}\right)_{x}+\left(v_{2, x}^{2}-v_{1, x}^{2}\right)-\left(w_{1}-w_{2}\right)_{x x}-2 v_{3, x x}=0
$$

where we have used the relations: $v_{1}+v_{2}=w_{1}-w_{2}+2 v_{3}$.

$$
E_{2}=\left(v_{1}+v_{2}\right)_{x t}-\left(v_{2}-v_{1}\right)_{x x x}-\left(v_{2}-v_{1}\right)_{x}\left(w_{1}+w_{2}\right)_{x x}-\left(2 v_{3}-v_{1}-v_{2}\right)_{x}\left(v_{1}+v_{2}\right)_{x x}=0,
$$

where we have used the relations: $v_{3}-v_{4}=2 v_{3}-v_{1}-v_{2}$. 
Eq.(4.6) can be decoupled into the following bilinearizable constraints:

$$
\begin{aligned}
& \mathcal{Y}_{t}\left(v_{1}, w_{1}\right)+\mathcal{Y}_{x x}\left(v_{1}, w_{1}\right)=0 \\
& \mathcal{Y}_{t}\left(v_{2}, w_{2}\right)+\mathcal{Y}_{x x}\left(v_{2}, w_{2}\right)=0 \\
& \mathcal{Y}_{x}\left(v_{3}, w_{3}\right)-\mu e^{v_{1}-v_{2}}=0
\end{aligned}
$$

Differentiating the first two equations of Eqs.(4.8) with respect to $x$ and using the relation $w_{1}+w_{2}+v_{2}-v_{1}=2 w_{3}$, Eq. (4.7) can be recasted in the form

$$
w_{3,3 x}+\left(v_{2}-v_{1}\right)_{x} w_{3,2 x}+v_{3, x}\left(v_{3}+v_{4}\right)_{x x}=0
$$

on account of Eq.(4.8c), the above expression amounts to the condition

$$
v_{3, x}\left(\frac{w_{3,2 x}}{v_{3, x}}\right)_{x}=-v_{3, x}\left(v_{3}+v_{4}\right)_{x x}
$$

which is satisfied if

$$
\begin{aligned}
& \frac{w_{3,2 x}}{v_{3, x}}+\left(v_{3}+v_{4}\right)_{x}=\gamma \Leftrightarrow \\
& \mathcal{Y}_{x x}\left(v_{3}, w_{3}\right)-\gamma \mathcal{Y}_{x}\left(v_{3}, w_{3}\right)+\mu e^{v_{1}-v_{2}} \mathcal{Y}_{x}\left(v_{4}, w_{4}\right)=0,
\end{aligned}
$$

so, according to Eq.(2.4) and the relations (4.2), Eqs.(4.8) and Eq.(4.9) can be transformed to Eqs.(4.1) for Eqs. (1.1).

\subsection{Lax pair}

Through the relations (4.2), we can get the following relations:

$$
v_{3}=v_{2}+p, \quad v_{4}=v_{1}-p, \quad w_{2}=w_{3}+p, \quad w_{4}=w_{1}+p .
$$

Setting that $w_{i}=v_{i}+Q_{i}, v_{i}=\ln \Psi_{i}(i=1,3)$, we can get

$$
Q_{1}=w_{1}-v_{1}=Q_{3}=w_{3}-v_{3}=q-p
$$

From Eq.(4.8c), Eq.(4.9) and the relations (4.10), we can get

$$
\Psi_{1, x}=\frac{\gamma}{2} \Psi_{1}-\frac{(q-p)_{x x}}{2 \mu e^{p}} \Psi_{3},
$$

from the BT Eq.(4.1c), we have

$$
\Psi_{3, x}=\mu e^{p} \Psi_{1},
$$

so, in combination with Eq.(4.11) and Eq.(4.12), we have

$$
\Psi_{x}=\left(\begin{array}{cc}
\frac{\gamma}{2} & -\frac{(q-p)_{x x}}{2 \mu e^{p}} \\
\mu e^{p} & 0
\end{array}\right) \Psi=M \Psi,
$$


where $\Psi=\left(\begin{array}{c}\Psi_{1} \\ \Psi_{3}\end{array}\right)$.

Again, from Eq.(4.8a) and Eq.(4.8b), we can get directly

$$
\Psi_{t}=N \Psi
$$

with

$$
\begin{aligned}
N & =\left(\begin{array}{cc}
\frac{-\gamma^{2}-2(q-p)_{x x}}{4} & \frac{\gamma(q-p)_{x x}-2 p_{x}(q-p)_{x x}+2(q-p)_{x x x}}{4 \mu e^{p}} \\
\frac{\mu e^{p}\left(2 p_{x}-\gamma\right)}{2} & \frac{2 p_{t}-2 p_{x x}^{2}-q_{x x}}{2}
\end{array}\right) \\
& =\left(\begin{array}{cc}
\frac{-\gamma^{2}-2(q-p)_{x x}}{4} & \frac{\gamma(q-p)_{x x}-2 p_{x}(q-p)_{x x}+2(q-p)_{x x x}}{4 \mu e^{p}} \\
\frac{\mu e^{p}\left(2 p_{x}-\gamma\right)}{2} & \frac{(q-p)_{x x}}{2}
\end{array}\right) .
\end{aligned}
$$

$M$ and $N$ are just the Lax pair of Eqs. (1.1), but they are very complicated, we will simplify it by two transformations.

Let $\psi=e^{p}, \varphi=-\frac{(q-p)_{x x}}{2 e^{p}}, M$ and $N$ are further transformed to

$$
M=\left(\begin{array}{cc}
\frac{\gamma}{2} & \frac{\varphi}{\mu} \\
\mu \psi & 0
\end{array}\right), \quad N=\left(\begin{array}{cc}
\frac{-\gamma^{2}}{4}+\psi \varphi & \frac{1}{\mu}\left(-\varphi_{x}-\frac{\gamma}{2} \varphi\right) \\
\mu\left(\psi_{x}-\frac{\gamma}{2} \psi\right) & -\psi \varphi
\end{array}\right) .
$$

In particular, if we let $\gamma=4 \lambda$, and

$$
\Psi=\left(\begin{array}{c}
\Psi_{1} \\
\Psi_{3}
\end{array}\right)=\left(\begin{array}{cc}
0 & \frac{\phi}{\mu} \\
\phi & 0
\end{array}\right)\left(\begin{array}{l}
\chi_{1} \\
\chi_{2}
\end{array}\right)
$$

where $\phi=e^{\lambda x-2 \lambda^{2} t}$, and $\lambda$ is an arbitrary real constant, then we can get

$$
\chi_{x}=\left(\begin{array}{l}
\chi_{1} \\
\chi_{2}
\end{array}\right)_{x}=\left(\begin{array}{cc}
-\lambda & \psi \\
\varphi & \lambda
\end{array}\right)\left(\begin{array}{l}
\chi_{1} \\
\chi_{2}
\end{array}\right)=\bar{M}\left(\begin{array}{l}
\chi_{1} \\
\chi_{2}
\end{array}\right),
$$

and

$$
\chi_{t}=\left(\begin{array}{l}
\chi_{1} \\
\chi_{2}
\end{array}\right)_{t}=\left(\begin{array}{cc}
2 \lambda^{2}-\psi \varphi & \psi_{x}-2 \lambda \psi \\
-\varphi_{x}-2 \lambda \varphi & -2 \lambda^{2}+\psi \varphi
\end{array}\right)\left(\begin{array}{l}
\chi_{1} \\
\chi_{2}
\end{array}\right)=\bar{N}\left(\begin{array}{c}
\chi_{1} \\
\chi_{2}
\end{array}\right) .
$$

$\bar{M}$ and $\bar{N}$ are also the Lax pair of Eqs. (1.1). When $u=(\ln \psi)_{x}, v=-\psi \varphi+(\ln \psi)_{x x}$, we can verify that the Levi equation (1.1) can be recovered by the compatibility condition $\bar{M}_{t}-\bar{N}_{x}+[\bar{M}, \bar{N}]=0$.

\section{Darboux transformation}

In what follows, we are going to discuss the Darboux transformation $[28,29]$ of the spectral problems (4.17) and (4.18). Now we introduce a gauge transformation

$$
\bar{\chi}=T \chi
$$


where $T$ is defined by

$$
\begin{aligned}
& T_{x}+T \bar{M}=\overline{\bar{M}} T, \\
& T_{t}+T \bar{N}=\overline{\bar{N}} T .
\end{aligned}
$$

The Lax pair (4.17) and (4.18) can be transformed into

$$
\bar{\chi}_{x}=\overline{\bar{M}} \bar{\chi}, \quad \bar{\chi}_{t}=\overline{\bar{N}} \bar{\chi}
$$

where $\overline{\bar{M}}$ and $\overline{\bar{N}}$ have the same forms as $\bar{M}$ and $\bar{N}$, respectively, except replacing $\psi$ and $\varphi$ with $\bar{\psi}$ and $\bar{\varphi}$.

Let the matrix $T$ be in the form of

$$
T=T(\lambda)=\left(\begin{array}{ll}
A & B \\
C & D
\end{array}\right)
$$

where

$$
\begin{aligned}
A & =\lambda^{N}+\sum_{k=0}^{N-1} \lambda^{k} A_{k}, & B & =\sum_{k=0}^{N-1} \lambda^{k} B_{k}, \\
C & =\sum_{k=0}^{N-1} \lambda^{k} C_{k}, & D & =\lambda^{N}+\sum_{k=0}^{N-1} \lambda^{k} D_{k},
\end{aligned}
$$

with $A_{k}, B_{k}, C_{k}$ and $D_{k}$ are the functions of $x$ and $t$, they can be determined by the following linear algebraic system:

$$
\sum_{k=0}^{N-1} \lambda_{j}^{k}\left(A_{k}+B_{k} \sigma_{j}\right)=-\lambda_{j}^{N}, \quad \sum_{k=0}^{N-1} \lambda_{j}^{k}\left(C_{k}+D_{k} \sigma_{j}\right)=-\sigma_{j} \lambda_{j}^{N},
$$

where

$$
\sigma_{j}=\frac{\zeta_{2}\left(\lambda_{j}\right)-r_{j} \eta_{2}\left(\lambda_{j}\right)}{\zeta_{1}\left(\lambda_{j}\right)-r_{j} \eta_{1}\left(\lambda_{j}\right)},(1 \leq j \leq 2 N)
$$

here $\zeta=\left(\zeta_{1}, \zeta_{2}\right)^{T}$ and $\eta=\left(\eta_{1}, \eta_{2}\right)^{T}$ are two basic solutions of the spectral problems (4.17) and (4.18), $\lambda_{j}$ and $r_{j}\left(\lambda_{k} \neq \lambda_{j}, r_{k} \neq r_{j}\right.$ as $\left.k \neq j\right)$ are some suitable parameters such that the determinant of the coefficients for Eq.(5.5) is nonzero. Therefore, $A_{k}, B_{k}, C_{k}$ and $D_{k}(0 \leq k \leq N-1)$ are uniquely determined by Eq.(5.5).

Theorem 5.1 The transformation from the old potentials $\psi$ and $\varphi$ into the new ones $\bar{\psi}$ and $\bar{\varphi}$ are given by

$$
\bar{\psi}=\psi+2 B_{N-1}, \quad \bar{\varphi}=\varphi-2 C_{N-1},
$$

then the matrices $\overline{\bar{M}}$ and $\overline{\bar{N}}$ have the same forms as $\bar{M}$ and $\bar{N}$, respectively. 
Proof: Let $T^{-1}=T^{*} / \operatorname{det} T$ and

$$
\left(T_{x}+T \bar{M}\right) T^{*}=\left(\begin{array}{ll}
f_{11}(\lambda) & f_{12}(\lambda) \\
f_{21}(\lambda) & f_{22}(\lambda)
\end{array}\right),
$$

where $T^{*}$ denotes the adjoint matrix of $T$. it is easy to see that $f_{11}(\lambda), f_{22}(\lambda)$ are $2 N+1$ th-order polynomials in $\lambda$, while $f_{12}(\lambda), f_{21}(\lambda)$ are $2 N$ th-order polynomials in $\lambda$. From the spectral problems $(4.17),(4.18)$ and the expression (5.6), we have a Riccati equation

$$
\sigma_{j x}=\varphi+2 \lambda_{j} \sigma_{j}-\psi \sigma_{j}^{2}, \quad \sigma_{j t}=\left(-\varphi_{x}-2 \lambda_{j} \varphi\right)-2\left(2 \lambda_{j}^{2}-\psi \varphi\right) \sigma_{j}-\left(\psi_{x}-2 \lambda_{j} \psi\right) \sigma_{j}^{2} .
$$

Through direct calculations, all $\lambda_{j}(1 \leq j \leq 2 N)$ are roots of $f_{s l}(s, l=1,2)$, therefore, Eq.(5.8) gives

$$
\left(T_{x}+T \bar{M}\right) T^{*}=(\operatorname{det} T) P(\lambda)
$$

with

$$
P(\lambda)=\left(\begin{array}{cc}
P_{11}^{(1)} \lambda+P_{11}^{(0)} & P_{12}^{(0)} \\
P_{21}^{(0)} & P_{22}^{(1)} \lambda+P_{22}^{(0)}
\end{array}\right),
$$

where $P_{k j}^{(l)}(k, j=1,2, l=0,1)$ are independent of $\lambda$. Now Eq.(5.8) can be written as

$$
\left(T_{x}+T \bar{M}\right)=P(\lambda) T
$$

By comparing the coefficients of $\lambda^{N+1}, \lambda^{N}, \lambda^{N-1}$ in (5.10), we find

$$
\begin{array}{ll}
P_{11}^{(1)}=-1, & P_{11}^{(0)}=0, \\
P_{12}^{(0)}=\psi+2 B_{N-1}, & P_{21}^{(0)}=\varphi-2 C_{N-1}, \\
P_{22}^{(1)}=1, & P_{22}^{(0)}=0,
\end{array}
$$

noticing the transformation (5.7), we see that

$$
P(\lambda)=\left(\begin{array}{cc}
-\lambda & \bar{\psi} \\
\bar{\varphi} & \lambda
\end{array}\right)=\overline{\bar{M}}
$$

Similarly,

$$
\left(T_{t}+T \bar{N}\right) T^{*}=(\operatorname{det} T) Q(\lambda)
$$

with

$$
Q(\lambda)=\left(\begin{array}{cc}
g_{11}^{(1)} \lambda^{2}+g_{11}^{(0)} & g_{12}^{(1)} \lambda+g_{12}^{(0)} \\
g_{21}^{(1)} \lambda+g_{21}^{(0)} & g_{22}^{(1)} \lambda^{2}+g_{22}^{(0)}
\end{array}\right)
$$

where $g_{k j}^{(l)}(k, j=1,2, l=0,1)$ are independent of $\lambda$. Now Eq.(5.11) can be written as

$$
\left(T_{t}+T \bar{N}\right)=Q(\lambda) T
$$


By comparing the coefficients of $\lambda^{N+2}, \lambda^{N+1}, \lambda^{N}$ in Eq.(5.12), we find

$$
\begin{array}{lll}
g_{11}^{(1)}=2, & g_{11}^{(0)}=-\left(\psi+2 B_{N-1}\right)\left(\varphi-2 C_{N-1}\right), \\
g_{12}^{(1)}=-2\left(\psi+2 B_{N-1}\right), & g_{12}^{(0)}=\psi_{x}-4 B_{N-2}-2 \psi\left(A_{N-1}-D_{N-1}\right)+4 B_{N-1} D_{N-1}, \\
g_{21}^{(1)}=-2\left(\varphi-2 C_{N-1}\right), & g_{21}^{(0)}=-\varphi_{x}+4 C_{N-2}+2 \varphi\left(A_{N-1}-D_{N-1}\right)-4 A_{N-1} C_{N-1}, \\
g_{22}^{(1)}=-2, & g_{22}^{(0)}=\left(\psi+2 B_{N-1}\right)\left(\varphi-2 C_{N-1}\right) .
\end{array}
$$

Comparing the coefficients of $\lambda^{N-1}$ in Eq.(5.10), we have

$$
\begin{aligned}
& B_{N-1, x}=-2 B_{N-2}-\psi\left(A_{N-1}-D_{N-1}\right)+2 B_{N-1} D_{N-1}, \\
& C_{N-1, x}=2 C_{N-2}+\varphi\left(A_{N-1}-D_{N-1}\right)-2 A_{N-1} C_{N-1},
\end{aligned}
$$

noticing the transformation (5.7), we obtain

$$
Q(\lambda)=\left(\begin{array}{cc}
2 \lambda^{2}-\bar{\psi} \bar{\varphi} & -2 \bar{\psi} \lambda+\bar{\psi}_{x} \\
-2 \bar{\varphi} \lambda-\bar{\varphi}_{x} & -2 \lambda^{2}+\bar{\psi} \bar{\varphi}
\end{array}\right)=\overline{\bar{N}}
$$

The proof is completed.

\section{$6 \quad$ Infinite conservation laws}

In this section, we derive the infinite conservation laws [30] for Eqs.(1.1) through the Lax equations (4.17) and (4.18).

Let $w(x, \lambda)=\frac{\chi_{2}}{\chi_{1}}$, Eq.(4.17) is transformed to

$$
w(x, \lambda)_{x}=-\psi w(x, \lambda)^{2}+2 \lambda w(x, \lambda)+\varphi
$$

or

$$
\psi w(x, \lambda)_{x}=-\psi^{2} w(x, \lambda)^{2}+2 \lambda \psi w(x, \lambda)+\psi \varphi
$$

inserting expansion

$$
\psi w(x, \lambda)=\sum_{n=0}^{\infty} w_{n}(x)(2 \lambda)^{-(n+1)}
$$

into Eq.(6.1), and comparing the coefficients of $(2 \lambda)^{-(n+1)}(n=0,1,2,3, \ldots)$, we get

$$
\begin{aligned}
& w_{0}(x)=-\psi \varphi=v-u_{x} \\
& w_{1}(x)=-\psi \varphi_{x}=v_{x}-u_{x x}-u v+u u_{x}, \\
& w_{n+1}(x)=\psi\left(\frac{w_{n}(x)}{\psi}\right)_{x}+\sum_{j=0}^{n-1} w_{j}(x) w_{n-j-1}(x) \quad(n=1,2,3, \ldots) .
\end{aligned}
$$


This is the formular of the coefficients of Eq.(6.2). Dividing both sides of the first equation of the Lax equations (4.17) and (4.18) by $\chi_{1}$, we get

$$
\begin{aligned}
& \left(\ln \chi_{1}\right)_{x}=-\lambda+\psi w(x, \lambda), \\
& \left(\ln \chi_{1}\right)_{t}=2 \lambda^{2}-\psi \varphi+\left(\psi_{x}-2 \lambda \psi\right) w(x, \lambda) .
\end{aligned}
$$

From the compatibility condition $\left(\ln \chi_{1}\right)_{t x}=\left(\ln \chi_{1}\right)_{x t}$, we have

$$
(-\lambda+\psi w(x, \lambda))_{t}=\left(2 \lambda^{2}-\psi \varphi+\left(\psi_{x}-2 \lambda \psi\right) w(x, \lambda)\right)_{x}
$$

that conservation laws is just

$$
(\psi w(x, \lambda))_{t}=\left(2 \lambda^{2}-\psi \varphi+\left(\psi_{x}-2 \lambda \psi\right) w(x, \lambda)\right)_{x} .
$$

The first conservation laws and the $n$th conservation laws are

$$
\begin{gathered}
\left(w_{0}(x)\right)_{t}=(\psi \varphi)_{t}=\left(\psi_{x} \varphi-\psi \varphi_{x}\right)_{x} \\
\left(w_{n}(x)\right)_{t}=\left(-w_{n+1}(x)+\frac{\psi_{x}}{\psi} w_{n}(x)\right)_{x}, \quad n=1,2,3, \ldots
\end{gathered}
$$

\section{Conclusions}

In this paper, we investigated the Levi equation (1.1) by using the binary Bell polynomials approach and Hirota method. Based on the bilinear equations for Eqs.(1.1), the double Wronskian solutions, the bilinear Bäcklund transformation, the corresponding Lax pair, the Darboux transformation and the infinite conservation laws of Eqs.(1.1) are all derived. These results show, combining the binary Bell polynomials approach and Hirota method not only can get more integrability properties of a nonlinear equation but also can avoid a lot of difficulties.

\section{Acknowledgements}

This work were supported in part by the National Science Foundation of China (nsfc(11202161) and sponsored by the Seed Foundation of Innovation and Creation for Graduate Students in Northwestern Polytechnical University (Grant No. Z2016165).

\section{References}

[1] M.J. Ablowitz and H. Segur, Solitons and the Inverse Scattering Transform, SIAM, Philadelphia (1981).

[2] M.R. Miura, Bäcklund Transformation, Springer-Verlag, Berlin (1978). 
[3] V.B. Matveev and M.A. Salle, Darboux Transformations and Solitons, Springer, Berlin (1991).

[4] R. Hirota, Exact solution of the Korteweg-de Vries equation for multiple collisions of solitons, Phys. Rev. Lett., 27 (1971) 1192.

[5] R. Hirota, the Direct Method in Soliton Theory, Cambridge Univ. Press, Cambridge (2004).

[6] Freeman N.C., Nimmo J.J., Soliton solutions of the Korteweg-de Vries and Kadomtsev-Petviashvili equations: the Wronskian technique, Phys. Lett. A, 95 (1983) 1.

[7] E.T. Bell, Exponential polynomials, Ann. Math., 35 (1934) 258.

[8] Gilson, C., Lambert, F., Nimmo, J., Willox, R., On the combinatorics of the Hirota D-operators. Proc. R. Soc. Lond. A, 452 (1996) 223.

[9] F. Lambert and J. Springael, On a direct procedure for the disclosure of Lax pairs and Bäcklund transformations, Chaos Soliton Fractals, 12 (2001) 2821.

[10] F. Lambert and J. Springael, Soliton Equations and Simple Combinatorics, Acta Appl. Math., 102 (2008) 147.

[11] E.G. Fan, The integrability of nonisospectral and variable-coefficient KdV equation with binary Bell polynomials, Phys. Lett. A, 375 (2011) 493.

[12] Y.H. Wang, Y. Chen, Binary Bell polynomial manipulations on the integrability of a generalized (2+1)-dimensional Korteweg-de Vries equation, J. Math. Anal. Appl., 400 (2013) 624.

[13] Z.J. Qiao and E.G. Fan, Negative-order Korteweg-de Vries equations, Phys. Rev. E, 86 (2012) 016601.

[14] Y. Jiang, B. Tian, W.J. Liu, M. Li, and P. Wang, Solitons, Bäcklund transformation, and Lax pair for the $(2+1)$-dimensional Boiti-Leon-Pempinelli equation for the water waves, J. Math. Phys., 51 (2010) 093519.

[15] P. Wang, B. Tian, W.J. Liu, X. Lu, Y. Jiang, Lax pair, Bäcklund transformation and multi-soliton solutions for the Boussinesq-Burgers equations from shallow water waves, Appl. Math. Comput., 218 (2011) 1726.

[16] Qin Y., Gao Y.T., Yu X., Meng G.Q., Bell Polynomial approach and N-soliton solutions for a coupled KdV-mKdV system, Commun. Theor. Phys., 58 (2012) 73 
[17] E.G. Fan and K.W. Chow, Darboux covariant Lax pairs and infinite conservation laws of the (2+1)-dimensional breaking soliton equation, J. Math. Phys., 52 (2011) 023504.

[18] X. Lü, B. Tian and F.H. Qi, Bell-polynomial construction of Bäcklund transformations with auxiliary independent variable for some soliton equations with one Tau-function, Nonlinear Analysis: Real Word Applications, 13 (2012) 1130.

[19] G.D. Lin, Y.T. Gao, Z.Y. Sun, X. Yu, D.X. Meng, Solitonic interactions and doubleWronskian-type solutions for a variable-coefficient variant Boussinesq model in the long gravity water waves, Appl. Math. Comput., 217 (2011) 4805.

[20] Y. Zhang, W.W. Wei, T.F. Cheng and Y. Song, Binary Bell polynomial application in generalized $(2+1)$-dimensional $\mathrm{KdV}$ equation with variable coefficients, Chin. Phys. B, 20 (2011) 110204.

[21] W.X. Ma, Bilinear Equations and Resonant Solutions Characterized by Bell Polynomials, Reports on Mathematical Physics, 72 (2013) 41.

[22] You F.C., Zhang J., Hao H.H., Multi-Soliton Solutions of the Levi Equations, Chin. Phys. Lett., 26 (2009) 090201

[23] L. Wang, Y.T. Gao, X.L. Gai and Z.Y. Sun, Inelastic interactions and double Wronskian solutions for the Whitham-Broer-Kaup model in shallow water, Phys. Scr., 80 (2009) 065017

[24] G.D. Lin, Y.T. Gao, L. Wang, D.X. Meng, X.Yu, Elastic-inelastic-interaction coexistence and double Wronskian solutions for the Whitham-Broer-Kaup shallow-waterwave model, Commun. Nonlinear Sci. Numer. Simulat., 16 (2011) 3090.

[25] Chen D.Y., Zhang D.J. and Bi J.B., New double Wronskian solutions of the AKNS equation, Science in China Series A: Mathematics, 51 (2008) 55.

[26] T. Xu, Y. Zhang, Fully resonant soliton interactions in the Whitham-Broer-Kaup system based on the double Wronskian solutions, Nonlinear Dyn., 73 (2013)485.

[27] Y. Zhang and D.Y. Chen, A modified Bäcklund transformation and multi-soliton solution for the Boussinesq equation, Chaos Soliton Fractals, 23 (2005) 175.

[28] X.M Li, A.H. Chen, Darboux transformation and multi-soliton solutions of Boussinesq-Burgers equation, Physics Letter A, 342 (2005)413.

[29] Zhaqilao, Darboux transformation and N-soliton solutions for a more general set of coupled integrable dispersionless system, Commun. Nonlinear Sci. Numer. Simulat., 16 (2011) 3949. 
[30] D.Y. Chen, Introduction of Soliton, Science Press, Beijing (2006). 